\title{
Prevalence and genotype distribution of group A rotavirus circulating in Shanxi Province, China during 2015-2019
}

Lifeng Zhao ${ }^{1}$, Xiaohong Shi², Dequan Meng ${ }^{1}$, Jiane Guo ${ }^{1}$, Yiping Li ${ }^{1}$, Lirong Liang ${ }^{1}$, Xiaofang Guo ${ }^{1}$, Ran Tao ${ }^{3}$, Xiaohua Zhang', Ruihong Gao', Li Gao ${ }^{1}$ and Jitao Wang ${ }^{1 *}$

\begin{abstract}
Background: Group A rotavirus (RVA), despite being a leading cause of gastroenteritis in infants and young children, is less studied in Shanxi Province, China. The current study was conducted to determine the prevalence and genetic characterization of RVA in hospitalized children younger than 10 years of age with the diagnosis of acute gastroenteritis in Shanxi Province, China.

Methods: A hospital-based active surveillance of rotavirus gastroenteritis was conducted at Children's Hospital of Shanxi from Jan 1, 2015, through Dec 31, 2019. Rotavirus was detected in stool samples by real-time quantitative reverse transcription PCR (qRT-PCR). G- and P-genotypes were determined by reverse transcription PCR (RT-PCR) and nucleotide sequencing.

Results: A total of 961 children younger than 10 years of age was enrolled over the study period, of whom 183 (19.0\%) were positive for RVA. The highest RVA-infection frequency (23.7\%) was found among children aged 12-23 months, and the seasonal peak was in December. G9P[8] was most prevalent (76.0\%), followed by G3P[8] (7.1\%), G2P[4] (3.3\%), G1P[8] (0.5\%) and G9P[4] (0.5\%).

Conclusions: These results report for the first time that RVA was one of the main causes of severe infectious gastroenteritis in children, and a high proportion of G9P[8] strains circulating in most areas of Shanxi Province. While the protective efficacy of the rotavirus vaccines has been demonstrated against G9P[8] strains, our results highlight that the dominant strains have not been effectively controlled in China.
\end{abstract}

Keywords: Group a rotavirus, Active surveillance, Acute gastroenteritis, Genotypes

\section{Background}

Group A rotaviruses (RVAs) are the leading etiological agents of severe childhood gastroenteritis worldwide [1]. The virus is a nonenveloped and double-stranded RNA virus belongs to the Reoviridae family. Members of the genus Rotavirus encoding six structural proteins (VP1-

\footnotetext{
* Correspondence: wangjitao99@126.com

'Department of Microbiology Test, Taiyuan Center for Disease Control and Prevention, NO. 89 Xinjian South Road, Taiyuan 030012, Shanxi Province, China

Full list of author information is available at the end of the article
}

VP4, VP6, VP7) and six nonstructural proteins (NSP1NSP6). Two outer capsid proteins, VP7 (or G protein) and VP4 (or P protein) are considered to define the serotype; their coding genes are classified into multiple genotypes based on sequence diversity [2, 3]. Currently, more than $36 \mathrm{G}$ and $51 \mathrm{P}$ types have been detected and at least 60 combinations have been identified from humans and other animals [4-6]. Globally, the most common G-P genotype combinations causing $90 \%$ infections in children were G1P[8], G2P[4], G3P[8], G4P[8],

(c) The Author(s). 2021 Open Access This article is licensed under a Creative Commons Attribution 4.0 International License, which permits use, sharing, adaptation, distribution and reproduction in any medium or format, as long as you give appropriate credit to the original author(s) and the source, provide a link to the Creative Commons licence, and indicate if changes were made. The images or other third party material in this article are included in the article's Creative Commons licence, unless indicated otherwise in a credit line to the material. If material is not included in the article's Creative Commons licence and your intended use is not permitted by statutory regulation or exceeds the permitted use, you will need to obtain permission directly from the copyright holder. To view a copy of this licence, visit http://creativecommons.org/licenses/by/4.0/ The Creative Commons Public Domain Dedication waiver (http://creativecommons.org/publicdomain/zero/1.0/) applies to the data made available in this article, unless otherwise stated in a credit line to the data. 
G9P[8], and G12P[8] [2, 3]. Of these, their relative proportions may vary by year and region [7].

Viral gastroenteritis is a common infectious disease syndrome, causing a combination of nausea, vomiting, diarrhea, and abdominal pain [8]. Rotaviruses are the dominant pathogens and more than 95\% of rotavirus gastroenteritis (RVGE) are caused by RVA [9]. Worldwide, according to WHO estimates in 2013, RVA was the leading cause of gastroenteritis death among children $<5$ years old and caused $\sim 215,000$ deaths each year [10]. In China, a survey, based on 45 reports from 1994 through 2014, indicated that RVAs cause $\sim 40 \%$ and $30 \%$ of viral-diarrhea-related hospitalizations and outpatient visits, respectively [11]. Besides, gastroenteritis caused by RVA can be more severe than that caused by other diarrhea viruses (e.g., norovirus, astrovirus, sapovirus) [12]. In a hospital-based study, RVA-positive was associated with a higher proportion of abdominal pain, dehydration, fever, and a greater estimated rate of hospitalization than RVA-negative [13].

Given the prevalence and gravity, the rotavirus vaccines are expected to play a critical role in reducing the tremendous burden. In China, LuoTeWei, also known as LLR (Lanzhou Lamb Rotavirus vaccine, Lanzhou Institute of Biological Products, China), has long been the only used vaccine within the last two decades (20002018), which is an oral, live, attenuated rotavirus vaccine sourced from G10P[12] of sheep [14]. LuoTeWei is currently recommended in China as a single dose annually from 2 months to 3 years of age and a booster dose between 3 and 5 years [14]. At the end of 2018, RotaTeq (Merck Sharp \& Dohme Products, USA) was available nationally and licensed for vaccinating children aged 632 weeks in China [15]. The effectiveness of any rotavirus vaccine is still unknown due to the comprehensive and continuous surveillance has not been carried out in China.

Up to now, the Rotavirus Surveillance System (RSS) was established only in regional cities such as Beijing, Jiangsu, Sichuan, and Guangdong. In Beijing and Guangdong, rotavirus continuous surveillance all began from 1998, which includes monitoring rotavirus test-positive rates and strain genotypes among inpatients younger than 5 years with diarrhea in selected sentinel hospitals [16]. In Sichuan and Jiangsu, except for rotavirus surveillance in children, there have been reports of rotavirus strains in animals [17-19]. Shanxi Province, located in the middle-area of China, is one of the regions with a serious burden of gastroenteritis infectiosa [20], but RVGE has long been ignored because it is considered to be a vaccine-preventable and self-limiting disease. Up to now, the incidence of rotavirus-infection has not been counted in the Chinese Disease Surveillance System (CDSS), so the genetic and epidemiological information of rotavirus is still unclear locally. Moreover, some rotavirus vaccines have been reported to be highly efficacious in reducing severe disease in many countries [2123], but rotaviruses continue to evolve and novel strains continue to emerge, whether those are effective is still unknown.

In the current study, a hospital-based surveillance of rotavirus in pediatric inpatients has been first established in 2015 in Shanxi Province, China. The specific aims were to estimate the disease burden, genotype distribution, and thus inform targeted control measures and vaccine development.

\section{Methods}

\section{Study area}

Shanxi Province is located in the middle-area of China (latitude $34^{\circ} 34^{\prime}-40^{\circ} 44^{\prime} \mathrm{N}$ and longitude $110^{\circ} 14^{\prime}-114^{\circ} 33^{\prime}$ E), consisting of 11 cities (80 counties) and a population of about 37 million.

\section{Specimens}

Fecal specimens were collected from children $<10$ years old who were hospitalized for diarrhea in Children's Hospital of Shanxi (CHS) between Jan 1, 2015, and Dec 31,2019 . Oral informed consent was obtained from their patients or guardians. All enrolled children have obvious symptoms of gastroenteritis (defined as $\geq 3$ loose stools over a $24 \mathrm{~h}$ period), and only those who have not received any medications and medical tests before hospitalization were eligible for enrolment. Patients exceed the age and diagnosed with chronic diarrhea by clinicians were excluded from this study. Specimens were collected in a sterile sampling cup, keeping low temperature $\left(4^{\circ} \mathrm{C}\right)$, and were sent to the virus microbiology laboratory of Taiyuan Center for Disease Control and Prevention (Taiyuan CDC) for rotavirus nucleic acid testing within $24 \mathrm{~h}$. Upon receipt, each sample was allocated a unique laboratory code and entered into a gastroenteritis information database (Excel) in Taiyuan CDC.

\section{RNA extraction}

Fecal suspensions $(10 \%, \mathrm{w} / \mathrm{v})$ were prepared of $0.01 \mathrm{M}$ phosphate-buffered saline (PBS) $(\mathrm{pH}=7.2)$, vortexed, and centrifuged $\left(8000 \mathrm{~g}\right.$ at $4{ }^{\circ} \mathrm{C}$ for $\left.8 \mathrm{~min}\right)$. Total RNA was extracted from $60 \mu \mathrm{L}$ of the fecal suspension using a MagMax-96 Viral RNA Isolation Kit (Thermo Fisher Scientific, Foster City, CA), according to the manufacturer's instructions. RNA-positive control and negative control (PBS) was included in the extraction procedure in each batch, and the quality of extracted RNA was checked through a NanoDrop 1000 Spectrophotometer (NanoDrop Technologies, Houston, TX, USA). Rotavirus nucleic acid testing and genotyping were kept physically 
separated. All steps of sample preparation and RNA extraction were done in a biosafety cabinet.

\section{Detection of rotavirus}

The real-time quantitative reverse transcription PCR (qRT-PCR) was applied by using a Rotavirus (Group A, B, and C) Multiple Real-time PCR Kit (TaqMan probe) (S-SBIO, Taizhou, China). PCR cycling parameters were set up according to the instruction: $50^{\circ} \mathrm{C}$ for $30 \mathrm{~min}$, $95^{\circ} \mathrm{C}$ for $5 \mathrm{~min}$, followed by 45 cycles of $94^{\circ} \mathrm{C}$ for $10 \mathrm{~s}$, $55^{\circ} \mathrm{C}$ for $40 \mathrm{~s}$ in a CFX96 Real-time Thermal Cycler (Bio-Rad, Hercules, CA). A positive result was defined as a threshold cycle $(\mathrm{Ct})$ value $<35$, and positive internal control was defined as a $\mathrm{Ct}$ value $<30$. The $\mathrm{qRT}$-PCR negative samples were not under the scope of our study.

\section{Gene amplification and nucleotide sequencing}

For VP7 amplification, the reverse transcription PCR was carried out using a PrimeScript One Step RT-PCR Kit. (TaKaRa, Dalian, China). A 1062-bp fragment was amplified with the consensus forward primer $B e g 9$ (GGCTTTAAAAGAGAGAATTTCCGTCTGG) and the reverse primer End9 (GGTCACATCATACAATTCTAAT $C T A A G)$. The amplification conditions included: $50^{\circ} \mathrm{C}$ for $30 \mathrm{~min}, 94^{\circ} \mathrm{C}$ for $2 \mathrm{~min}$ followed by 32 cycles of $94{ }^{\circ} \mathrm{C}$ for $30 \mathrm{~s}, 55^{\circ} \mathrm{C}$ for $30 \mathrm{~s}, 72^{\circ} \mathrm{C}$ for $90 \mathrm{~s}$, and a final extension of $72{ }^{\circ} \mathrm{C}$ for $5 \mathrm{~min}$. For the $V P 4$ amplification, the semi-nested reverse transcription PCR was carried out using a PrimeScript One Step RT-PCR Kit. The first amplification was carried out using primers VP4F2 (TTTATAGACAGCTTCTCACTAATTC) and VP4R3 (TATGTGCAGTTACTTGTTCACC) under the condition of $50^{\circ} \mathrm{C}$ for $30 \mathrm{~min}, 94^{\circ} \mathrm{C}$ for $2 \mathrm{~min}$, followed by 10 cycles of $94{ }^{\circ} \mathrm{C}$ for $30 \mathrm{~s}, 55^{\circ} \mathrm{C}$ for $30 \mathrm{~s}, 72{ }^{\circ} \mathrm{C}$ for $90 \mathrm{~s}$. Then $1 \mu \mathrm{l}$ of the first PCR products was used for amplification with the nested primers VP4F4 (TTTATAGACA GCTTCTCACTAATTC) and VP4R3 (TATGTGCAGT TACTTGTTCACC). The amplification conditions included $94{ }^{\circ} \mathrm{C}$ for $5 \mathrm{~min}$, followed by 30 cycles of $94{ }^{\circ} \mathrm{C}$ for $30 \mathrm{~s}, 55^{\circ} \mathrm{C}$ for $30 \mathrm{~s}, 72^{\circ} \mathrm{C}$ for $1 \mathrm{~min}$, and a final extension of $72^{\circ} \mathrm{C}$ for $5 \mathrm{~min}$. A portion ( $5 \mathrm{ul}$ ) of the reaction mixture with loading buffer, followed electrophoresis in $1.5 \%$ agarose gel (Takara, Dalian, China), was visualized by Goldview (Transgen, Beijing, China). The amplicons were purified and sequenced by Sangon Biotech (Shanghai, China). All the PCR runs included the positive control and non-target control (reagent blank) to avoid false-positive results. All the primers were synthesized by Sangon Biotech (Shanghai, China).

\section{Determination of RVA genotypes and phylogenetic analysis}

The resulting sequences were prepared and aligned by BioEdit (version 7.2.5) with the Clustal-W program.
Based on the sequence data, the genotype assignment was accomplished using BLAST (http://blast.ncbi.nlm. nih.gov/Blast.cgi) and RotaC v2.0 (http://rotac.regatools. be). Phylogenetic trees were constructed using the Neighbor-joining method with MEGA (version 5.0) and bootstrap analysis was performed with 1000 replications. The reference sequences used in plotting the phylogeny trees from the GenBank database are shown in Table 1.

\section{Nucleotide sequence accession numbers}

The nucleotide sequences used in this study were submitted in GenBank (https://www.ncbi.nlm.nih.gov/ genbank/) under accession numbers MT710743 MT710824 (VP7) and MT710825 - MT710927 (VP4), which are shown in Table S2.

\section{Statistical analysis}

Data analyses were performed by IBM SPSS Statistics software (Version 19.0). Univariate logistic regression was performed to ascertain the equality of means of RVA positive or negative for variables: sexes, symptoms, and sample types (Table 2). Statistical significance was defined as $p<0.05$.

\section{Results}

\section{Patients with gastroenteritis}

During the period 2015-2019, a total of 961 hospitalized children younger than 10 years of age, 609 boys and 352 girls, confirmed providing fecal specimens for rotavirus testing. RVA was detected in 183 stool samples via qRTPCR with an overall positivity rate of $19.0 \%$ (183/961). Rotavirus $\mathrm{B}$ and $\mathrm{C}$ were not detected. The maximum number of samples was tested in May 2017, and the largest number of RVA-positive cases occurred in February 2019 (Fig. 1). No statistically significant difference was found between sex and RVA-infection $(p>0.05)$ (Table 2 ). Types of fecal specimen collected in this study included watery stool, loose stool, pus and blood stool, and mucus stool, no statistically significant difference existed between four types positive for RVA (Table 2). Apart from the symptom of diarrhea, some patients were accompanied by other related symptoms such as vomiting, fever, abdominal pain, and dehydration. There was a statistically significant difference between RVA-infection and fever $\left(>38.5^{\circ} \mathrm{C}\right)(p<0.05)$. Whereas, no significant difference $(p>0.05)$ was found between RVA-infection and the other three symptoms (vomiting, abdominal pain, and dehydration) (Table 2). Twenty RVA-positive patients coinfected with other diarrhea associated viruses: 16 with norovirus, 2 with sapovirus, and 1 with astrovirus. One was coinfected with more than two viruses (norovirus and astrovirus). Six were coinfected with other pathogenic bacteria: 2 with Salmonella, 3 with EAEC, and 1 with ETEC (Table S1). 
Table 1 Reference sequences used in plotting the phylogenetic tree of this study

\begin{tabular}{|c|c|c|c|c|c|c|c|c|c|}
\hline $\begin{array}{l}\text { Accession } \\
\text { number }\end{array}$ & Strains & Date & Location & Genotypes & $\begin{array}{l}\text { Accession } \\
\text { number }\end{array}$ & Strains & Date & Location & Genotypes \\
\hline KU243671 & WZ202 & 2016 & Zhejiang (China) & G1 & MG816527 & SC6 & 2013 & Sichuan (China) & G9 \\
\hline KX009876 & Kerala-RV01 & 2013 & India & G1 & KX778608 & km15119 & 2016 & Yunnan (China) & G9 \\
\hline GU565057 & RotaTeq-WI79-9 & 1992 & USA & G1 & KT919508 & VU12-13-101 & 2013 & USA & G9 \\
\hline HM130956 & KR/Seoul-710 & 2009 & Korea & G2 & LC477377 & Tokyo18-43 & 2018 & Japan & G9 \\
\hline LC477357 & Tokyo17-10 & 2017 & Japan & G2 & AB180971 & $\mathrm{A} 2$ & $19 x x^{a}$ & USA & G9 \\
\hline GU565068 & RotaTeq-SC2-9 & 1992 & USA & G2 & KX363355 & 14,150 & 2012 & Vietnam & G9 \\
\hline KF371856 & E2432 & 2010 & Hubei (China) & G3 & MF139499 & CU192 & 2016 & Thailand & G9 \\
\hline LC477355 & Tokyo17-08 & 2017 & Japan & G3 & KU243609 & WZ189 & 2013 & Zhejiang (China) & $P[4]$ \\
\hline KY661928 & $1 C R 7$ & 2015 & Thailand & G3 & MG729831 & $\mathrm{Hu} / 13-146$ & 2013 & Shanghai (China) & $P[4]$ \\
\hline GU565079 & RotaTeq-WI78-8 & 1992 & USA & G3 & KF372017 & Z1602 & 2012 & Hubei (China) & $\mathrm{P}[8]$ \\
\hline EU348715 & P50 & $20 x x^{a}$ & Slovenia & G3 & KX778584 & Km15119 & 2016 & Yunnan (China) & $\mathrm{P}[8]$ \\
\hline KX911619 & CU140 & 2016 & Thailand & G3 & MG816520 & $\mathrm{SC} 1$ & 2014 & Sichuan (China) & $\mathrm{P}[8]$ \\
\hline L35055 & A131 & 1988 & Venezuela & G3 & MF580855 & $\mathrm{Hu} / J S 2012$ & 2012 & Jiangsu (China) & $\mathrm{P}[8]$ \\
\hline KF673479 & BJ-Q322 & 2011 & Beijing (China) & G9 & LC477409 & Tokyo18-50 & 2018 & Japan & $\mathrm{P}[8]$ \\
\hline KF673482 & BJ-Q794 & 2012 & Beijing (China) & G9 & GU565044 & RotaTeq-WI79-4 & 1992 & USA & $\mathrm{P}[8]$ \\
\hline MF580843 & $\mathrm{Hu} / \mathrm{JS} 2013$ & 2013 & Jiangsu (China) & G9 & & & & & \\
\hline
\end{tabular}

Note: ${ }^{\mathrm{a}}$ There are missing data

\section{Age, geographical and seasonal features}

Overall, most cases (292) were found in the age group of 12-23 months which was also the group with the highest average RVA-positive rate (23.7\%) (Fig. 2a). On the contrary, the lowest rate $(8.5 \%)$ was observed in the group > 60 months with a statistically significant difference $(p<$ 0.05, Fig. 2a). More than one-fifth of gastroenteritis cases were found in July-August, but the RVA-positive rate remained low and with a statistically significant difference
( $p<0.05$, Fig. 2b). $44.9 \%$ of the RVA-positive cases were found in December, although gastroenteritis cases were rare in the cold season (November-January) (Figs. 1 and $2 b)$. All the patients came from the cities or regions of Shanxi Province, the highest prevalence (29.5\%) was observed in Yangquan, followed by $22.4 \%$ in Xinzhou, $21.8 \%$ in Lvliang, $21.1 \%$ in Northern Shanxi, and $16.9 \%$ in Taiyuan (Fig. 2c). The patients from Southern Shanxi had the lowest average positive rate (11.6\%, Fig. 2c).

Table 2 Sexual distinction, symptoms, and sample types of this study

\begin{tabular}{|c|c|c|c|c|c|}
\hline Demographics & Total & RVA-positive (n) & RVA-negative ( $n$ ) & RVA-positive (\%) & $P$-value \\
\hline \multicolumn{6}{|l|}{ Gender } \\
\hline Male & 609 & 114 & 495 & 18.72 & \\
\hline Female & 352 & 69 & 283 & 19.60 & 0.46 \\
\hline \multicolumn{6}{|l|}{ Symptom (Diarrhea with) } \\
\hline vomiting & 213 & 51 & 162 & 23.9 & 0.13 \\
\hline fever & 403 & 92 & 311 & 22.8 & 0.02 \\
\hline abdominal pain & 38 & 3 & 35 & 7.9 & 0.13 \\
\hline dehydration & 17 & 3 & 14 & 17.6 & 0.61 \\
\hline \multicolumn{6}{|l|}{ Sample type } \\
\hline Watery stool & 663 & 145 & 518 & 21.9 & 0.84 \\
\hline Loose stool & 151 & 22 & 129 & 14.6 & 0.79 \\
\hline Purulent bloody stool & 74 & 8 & 66 & 10.8 & 0.59 \\
\hline Mucus stool & 67 & 7 & 60 & 10.4 & 0.54 \\
\hline
\end{tabular}




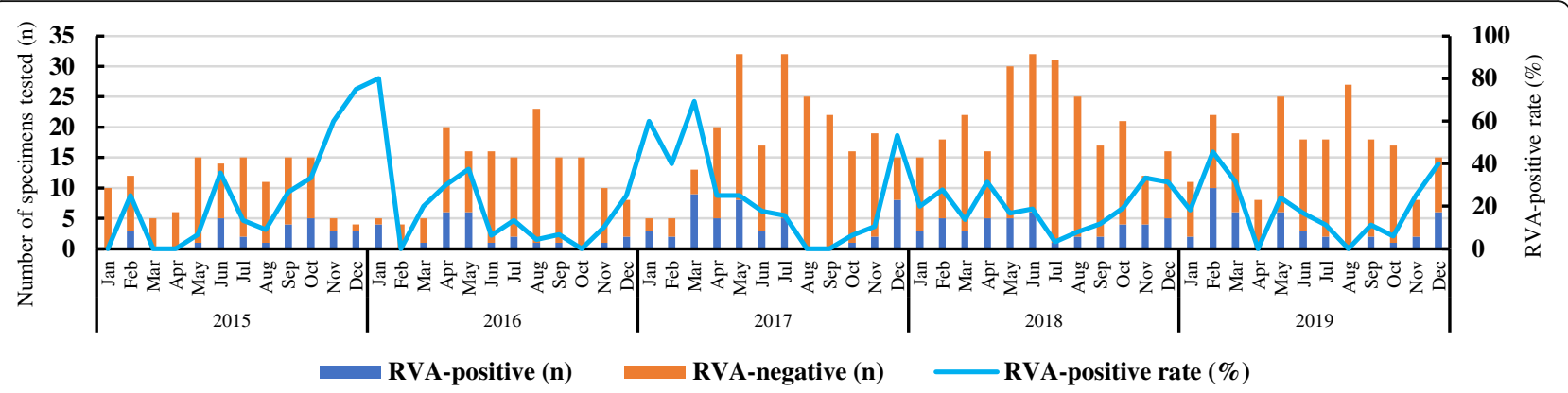

Fig. 1 Monthly distribution and rotavirus positivity among hospitalized children under ten years old with gastroenteritis in Shanxi Province, China, 2015-2019

\section{VP7 and VP4 genotyping}

One hundred fifty-nine samples were genotyped for $V P 7$, and 183 samples were successfully genotyped for VP4. G9 was the prevalent G-genotype, representing $76.0 \%(139 / 183)$ of all positive samples, followed by G3 (7.1\%, 13/183), G2 (3.3\%, 6/183), and G1 $(0.5 \%, 1 / 183)$ (Fig. 3a). P $[8](95.1 \%, 174 / 183)$ was the prevalent Pgenotype, followed by $\mathrm{P}$ [4] (4.9\%, 9/183) (Fig. 3b). One hundred fifty-nine strains were confirmed both G- and P-genotype. G9P[8] was the most commonly detected genotype $(76.0 \%, 139 / 183)$, followed by G3P[8] $(7.1 \%$, $13 / 183)$, G2P[4] (3.3\%, 6/183), G1P[8] (0.5\%, 1/183), and G9P[4] (0.5\%, 1/183) (Fig. 3c).

\section{Geographical distribution}

Due to the long distance between the patients and the designated hospital, cases from two northern cities (Datong and Shuozhou) and four southern cities (Linfen, Yuncheng, Changzhi, and Jincheng) were less compared with the central cities of Shanxi Province, therefore, cases from these cities were divided into two independent parts (Northern Shanxi and Southern Shanxi) for next analyzing. G9P[8] was the dominant genotype and occupied more than $80 \%$ of RVA positive cases in most cities or regions of Shanxi Province (Fig. 4). The only G1P[8] and G9P [4] were detected in Taiyuan (2016) and Southern Shanxi (2018), respectively (Fig. 4). G2P[4] was detected in Taiyuan, Xinzhou, Lvliang, and Yanquan; G3P[8] was detected in Taiyuan, Xinzhou, Lvliang, and Southern Shanxi. Strains that cannot be typed (GntP[8] and $\mathrm{GntP}[4])$ were found in most areas in Shanxi province, except Yangquan (Fig. 4).

\section{Phylogenetic analyses of VP7 genes}

The G1-G2-G3 VP7 tree was constructed based on 18 Shanxi strains sequenced in this study and 13 representative members (Fig. 5, Table 1). The G1 lineage contained the only Shanxi strain (SX/2016/073) and two representative strains (WZ202 and Kerala-RV01) detected in China and Indonesia with 95.9 and 99.8\% nucleotide similarity, respectively. SX/2016/073 shared $90.8 \%$ nucleotide similarity and $92.8 \%$ amino acid similarity with the RotaTeq G1 strain RotaTeq-WI79-9 (Fig. 5, G1). Five Shanxi G2 strains (SX/2015/136, SX/2017/ 267, SX/2019/270, SX/2014/140, and SX/2016/011) were closely related to a Japanese strain (Tokyo 17-10) detected in 2017, with $99.8-99.9 \%$ nucleotide similarities. The other Shanxi G2 strain (SX/2018/393) was related to a South Korea strain (Seoul-710) with a nucleotide similarity of $94.9 \%$. All the Shanxi G2 strains shared 92.7-92.9\% nucleotide similarity and $95.1-95.5 \%$ amino acid similarity with the RotaTeq G2 strain RotaTeqSC2-9 (Fig. 5, G2). Ten Shanxi G3 strains (SX/2017/ 001, SX/2017/021, SX/2017/080, SX/2017/172, SX/2017/ 020, SX/2017/473, SX/2017/082, SX/2017/047, SX/2018/ 089, and SX/2018/146) were closely related to the representative strains (E2432 and Tokyo 17-08) with high nucleotide similarities (99.3-99.6\%). The other Shanxi G3 strain (SX/2017/271) was related to 1CR7 detected in South Korea (2017), with 99.3\% nucleotide similarity. All the Shanxi G3 strains shared 81.8-94.1\% nucleotide similarity and $92.5-97.7 \%$ amino acid similarity with the RotaTeq vaccine G3 strain RotaTeq-SC2-9 (Fig. 5, G3).

Sixty-three Shanxi G9 strains and 10 representative members were selected for G9 phylogenetic analyses (Fig. 6, Table 1). Except for SX/2015/069, 62 Shanxi G9 strains fell into 2 minor lineages (I and II). Fifty-five Shanxi G9 strains (belong to lineage 1) were closely related to each other with $99.2-99.9 \%$ nucleotide similarity, clustering with other strains isolated in Japan (Tokyo 18-43), USA (VU12-13-101), Thailand (T152), and China (SC6, Hu/JS2013, BJ-Q794) during 2011-2018 (Fig. 6, lineage I). Seven Shanxi G9 strains (SX/2015/ 061, SX/2015/065, SX/2015/081, SX/2015/059, SX/2015/ 132, SX/2015/156, and SX/2016/002) belong to lineage II were closely related to each other with $99.4-99.9 \% \mathrm{nu}$ cleotide similarity, clustering with a Chinese strain (km15119) isolated in Yunnan Province in 2016 (Fig. 6, 
a

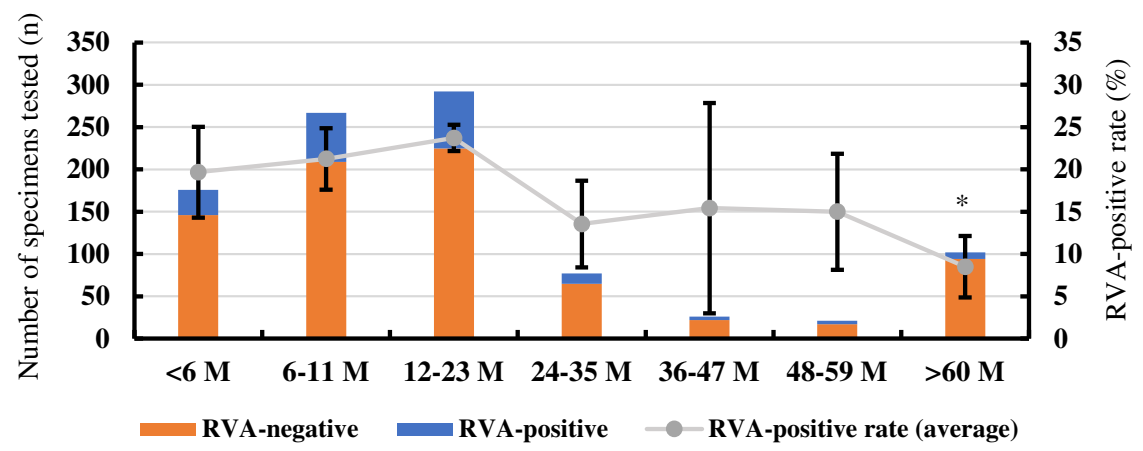

b

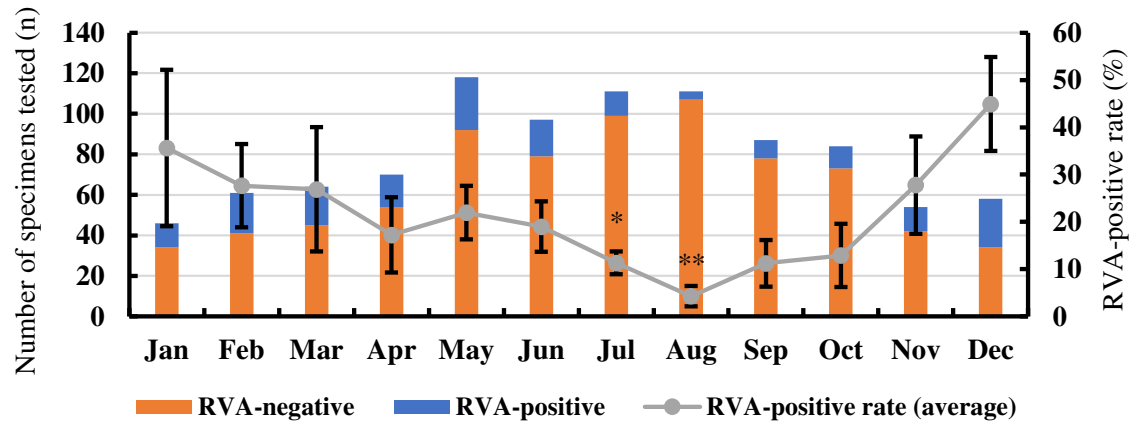

c

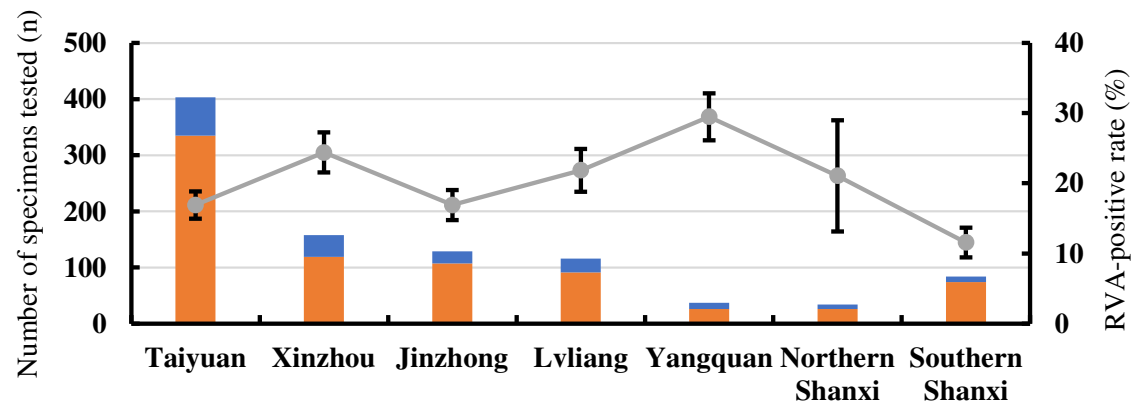

RVA-negative RVA-positive $\quad$ - RVA-positive rate (average)

Fig. 2 Prevalence of RVA infections in Shanxi Province, China, 2015-2019. a Age distribution; b Monthly distribution; c Geographical distribution. $P$ values: ${ }^{*} p<0.05,{ }^{* *} p<0.01$. Error bars: standard deviation

lineage II). The SX/2015/069 was separate from other Shanxi G9 strains, but still has 99.2\% nucleotide similarity with the reference strain HU/JS2013 (Fig. 6).

\section{Phylogenetic analyses of VP4 genes}

The P[4]-VP4 tree was constructed based on 9 Shanxi strains and 2 representative members (Fig. 7, Table 1). Eight Shanxi strains (SX/2015/136, SX/2019/271, SX/ 2019/284, SX/2018/400, SX/2019/270, SX/2016/041, SX/ $2017 / 267$, and $S X / 2015 / 140$ ) were closely related to WZ189 detected in Zhejiang Province (China) in 2017, with high nucleotide similarities (99.7-99.9\%). The other Shanxi strain SX/2018/393 was closely related to the
$\mathrm{Hu} / 13-146$ detected in Shanghai (China), with 98.0\% nucleotide similarity (Fig. 7).

The P[8]-VP4 tree was constructed based on 93 Shanxi strains and 6 representative members (Fig. 8, Table 1). The Shanxi G9 strains fell into 2 minor lineages (I and II, Fig. 8). Eighty-seven Shanxi P[8] strains (belong to lineage 1) were closely related to each other with 96.9-99.9\% nucleotide similarity, clustering with a Japanese strain (Tokyo 18-50) and 3 China's strains (Z1602, km15119, SC1) isolated in previous studies during 2011-2018. The Shanxi P[8] strains belong to lineage I shared $91.9-93.0 \%$ nucleotide similarity and $93.1-94.0 \%$ amino acid similarity 


\section{a}

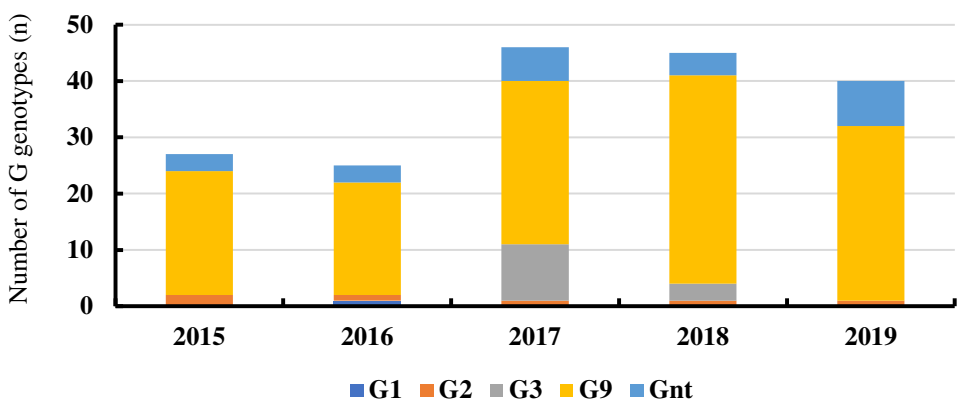

b

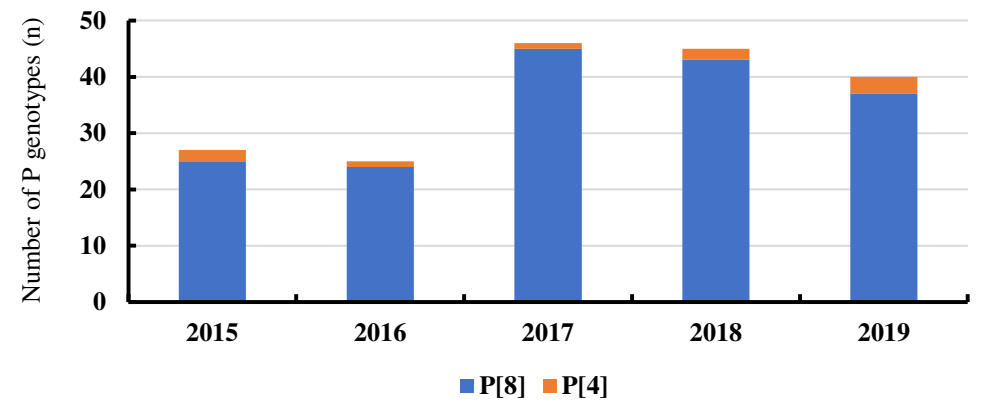

c

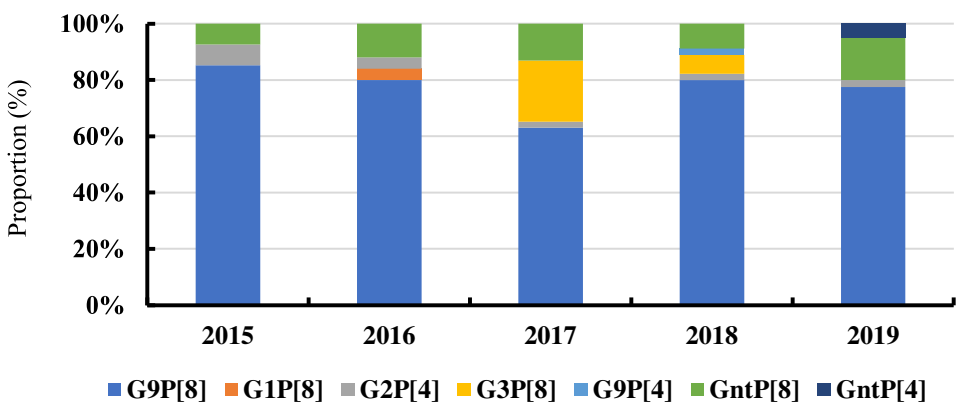

Fig. 3 Prevalence of different RVA genotypes by surveillance year in Shanxi province, China, 2015-2019. a G genotypes; b P genotypes; c Proportions of different genotypes

with the RotaTeq vaccine $\mathrm{P} 1 \mathrm{a}[8]$ strain RotaTeqWI79-4 (Fig. 8, lineage I). The other 6 Shanxi P[8] strains (SX/2016/124, SX/2016/172, SX/2016/112, SX/ 2015/144, SX/2015/117, and SX/2015/069) belong to lineage 2 were closely related to a Chinese strain $\mathrm{Hu} /$ JS-2012 detected in Jiangsu Province in 2012, with 98.9-99.3\% nucleotide similarities (Fig. 8, lineage II). The Shanxi P [8] strains belong to lineage II shared $87.6-87.7 \%$ nucleotide similarity and $88.4-88.8 \%$ amino acid similarity with the RotaTeq vaccine $\mathrm{P} 1 \mathrm{a}[8]$ strain RotaTeq-WI79-4 (Fig. 8).

\section{Discussion}

Infecting nearly every child, human group A rotaviruses have been proven to be a major cause of pediatric diarrhoeal disease morbidity and mortality all through the world [24].

In the current study, the RVA-positive rate found in children < 10 years old in Shanxi Province was 19.0\%, which is comparable with the results of 20.8 and $17.5 \%$ in Beijing (2011-2016, 15] and Chengdu (Sichuan Province, China, 2009-2014) [25], respectively. However, a sentinel-based surveillance of diarrhea study by Zeng in Chongqing found a prevalence of $30.5 \%$ in the period of 2011-2015 [13], which is much higher than the current study. There might be several reasons, such as different patient categories (outpatient or inpatients), different definitions of gastroenteritis, selection of suspected cases, types of the sample tested (serum, plasma, or feces), the standard for valid sample, and types of diagnostic reagent (ELISA or RT-PCR). Specifically, among 


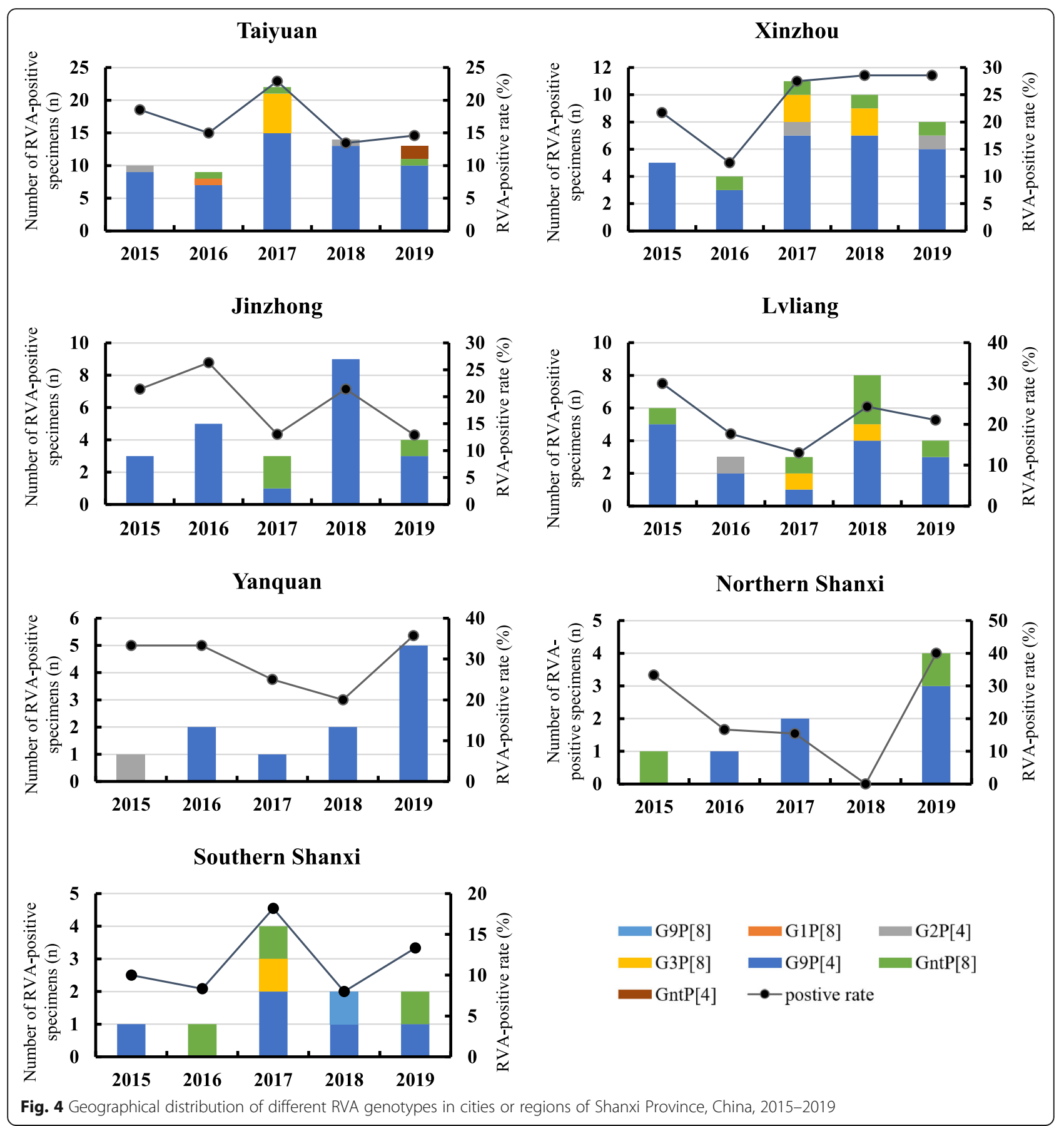

children under 5 years old, the positive rate in Shanxi Province is $20.4 \%(175 / 859)$. Compared with the reports from other WHO regions where rotavirus vaccines have been introduced after 2008, the average proportion of acute gastroenteritis cases positive for rotavirus in our study was lower than African Region, Region of the Americas, Eastern Mediterranean Region, and higher than European Region [26].

In this study, up to one-half of inpatients have febrile symptoms $\left(>38.5^{\circ} \mathrm{C}\right)$, and more than one quarter have a symptom of vomiting (Table 2). However, it is not possible to diagnose RVA infection by clinical presentation because the clinical features of RVGE do not differ from those caused by other pathogens. Severe, lethal RVA infections did not appear among all the RVA-positive cases, which may be different from Liu's research [11]. During the whole process, based on the data of routine blood tests, we found that the proportion of viral infections was significantly higher than bacterial infections (data not shown). However, the abuse of antibiotics has 


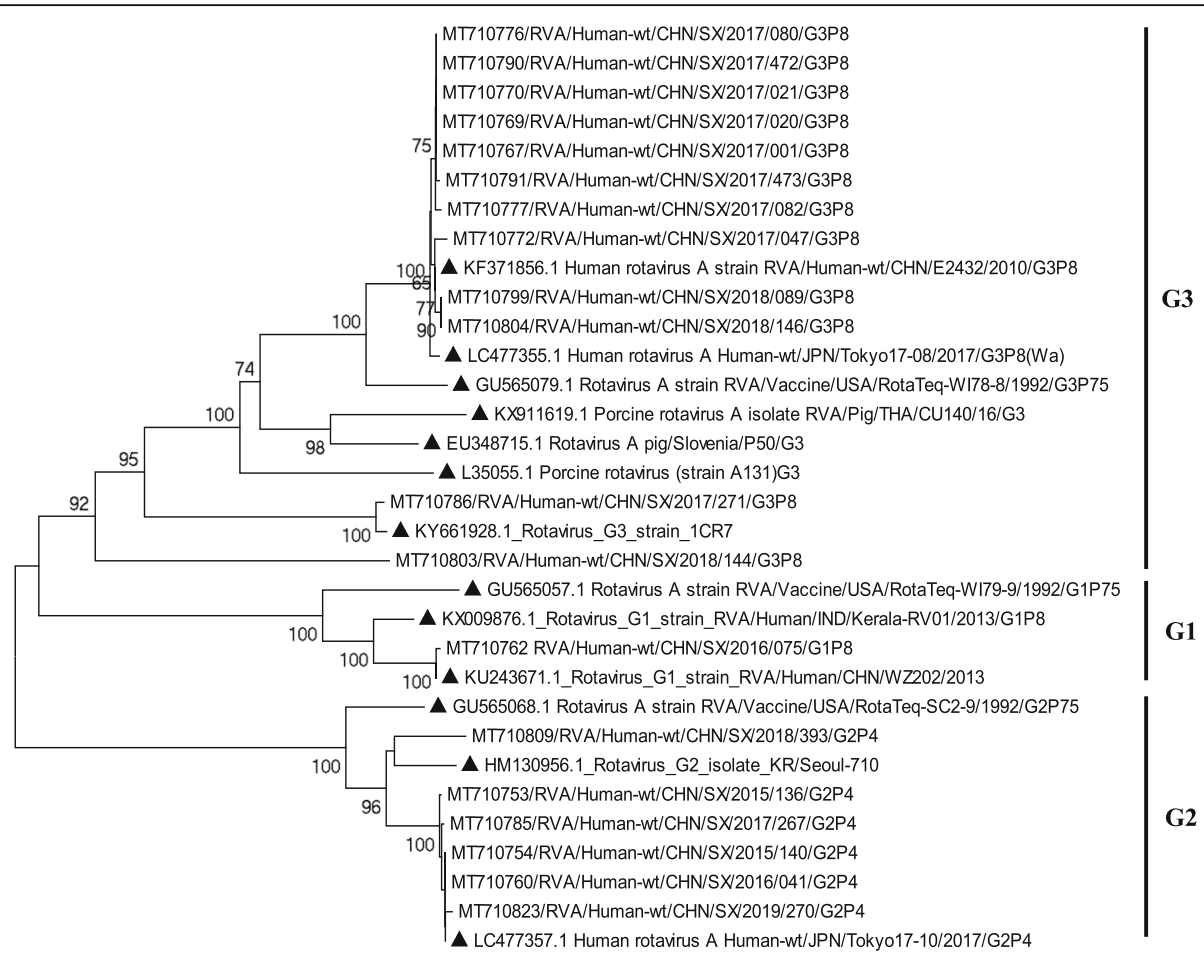

Fig. 5 Neighbor-joining phylogenetic tree constructed from the partial VP7 genes (798 bp) of G1, G2, and G3 strains, and representative RVA strains with the kimura-2-parameter model in MEGA program 5.0. Bootstrap values estimated with 1000 replicate data sets were indicated at each node. The scale bar indicated the number of nucleotide substitutions per site. Bootstrap values lower than $60 \%$ are not shown. The reference strains are represented by the triangle. The RVA strains sequenced in this study are represented by Accession number/Country/Province/Year/ Serial number/Genotype

not been effectively controlled in China, and most gastroenteritis patients have used antibiotics before seeing a doctor. Therefore, it is impossible to prove that virus is the main cause of child gastroenteritis compared with bacteria in Shanxi Province.

Available data suggest that RVA is susceptible to people of any age but spreading more easily among infants and young children [27]. Studies in Shanghai had discovered that the RVA prevalence was higher in children aged 3-5 years [28]. In our study, RVA-infection mainly occurs in children under 2 years of age with a peak positive rate in children of $12-23$ months, followed by $6-11$ months, and $0-6$ months (Fig. 2a), which was similar to a Beijing's study [15]. Considering the highest positive rate was in the $0-23$-month age group, early introduction of rotavirus vaccination to children is desirable.

Globally, there are obvious seasonal and geographical variations in RVA prevalence. In this study, RVA infections occurred throughout the year, but with the highest detection rate in the cold season (Fig. 2b). This finding is identical to a monitoring analysis by $\mathrm{Yu}$ et al. on the seasonality of RVA infection [29]. The same patterns have also been reported in other Asian countries like Pakistan [30] and India [27]. However, according to the WHO, RVA prevalence was reported throughout the year in the subtropical zone and peaks often occur in dry seasons, unlike in the northern hemisphere countries where peaks occur in winter [31]. In Benin, West Africa, a higher rate of RVA-infection was recorded during the rainy season and the long dry season [31].

Molecular epidemiology studies have revealed that the distribution of RVA strains varies over both space and time. In China, G1/G3/G9-P[8] and G2P[4] were most commonly reported in the epidemiological studies from 1994 to 2013 [15]. According to the existing research results, the formation of dominant genotypes is a longterm evolutionary process. In 2011, the genotype G3P [8] with G9P [8] was first found to be both the dominant genotypes in Beijing [15]. In 2015, Yu reported the predominant strain of RVA has rapidly evolved from G3P[8] and G1P[8] to G9P[8], with the proportion of G9P [8] having increased remarkably from 3.4\% (2009) to $60.9 \%$ (2015) [29]. However, our results are significantly different from those of other countries in the last 10 years. In India, a study about RVA diversity showed that 
65 MT710788/RVA/Human-wt/CHN/SX/2017/457/G9P8 MT710794/RVA/Human-wt/CHN/SX/2018/026/G9P8 MT710789/RVA/Human-wt/CHN/SX/2017/459/G9P8 - MT710775/RVA/Human-wt/CHN/SX/2017/071/G9P8 MT710743/RVA/Human-wt/CHN/SX/2015/011/G9P8 - MT710781/RVA/Human-wt/CHN/SX/2017/157/G9P8 MT710759/RVA/Human-wt/CHN/SX/2016/037/G9P8 -MT710783/RVA/Human-wt/CHN/SX/2017/163/G9P8 MT710798/RVA/Human-wt/CHN/SX/2018/087/G9P8 - MT10822/RVA/Human-wt/CHN/SX/2019/118/G9P8 - MT170822RVA/Human-W/CHN/SX/2019/118/G9P8 MT710801/RVA/Human-w/CHN/SX/2018/109/G9P8 MT710778/RVA/Human-wt/CHN/SX2017/114/G9P8 - MT710821/RVA/Human-wt/CHN/SX/2019/116/G9P8 [ MT710817/RVA/Human-wt/CHN/SX/2019/045/G9P8 MT710805/RVA/Human-wt/CHN/SX/2018/322/G9P8 4 MT10806/RVA/Human-wt/CHN/SX/2018/329/G9P8 MT710793/RVA/Human-wt/CHN/SX/2018/010/G9P8 A MF580843.1 Human rotavirus A isolate Hu/JS2013 MT10784/RVA/Human-wt/CHN/SX/2017/165/G9P8

A MG816527.1 Rotavirus A isolate human/SC6/CHN/2013/G9

MT710800/RVA/Human-wt/CHN/SX/2018/095/G9P8

- MT710766/RVA/Human-wt/CHN/SX/2016/384/G9P8

MT710780/RVA/Human-wt/CHN/SX2017/152/G9P8 MT710782/RVA/Human-wt/CHN/SX/2017/159/G9P8 MT710810/RVA/Human-wt/CHN/SX/2018/400/G9P4 MT710811/RVA/Human-wt/CHN/SX/2018/412/G9P8 MT710813/RVA/Human-wt/CHN/SX/2019/006/G9P8

90-MT710807/RVA/Human-wt/CHN/SX/2018/357/G9P8

- MT710814/RVA/Human-wt/CHN/SX/2019/013/G9P8 MT710820/RVA/Human-wt/CHN/SX/2019/102/G9P8 - MT710797/RVA/Human-wt/CHN/SX/2018/074/G9P8 MT710812/RVA/Human-wt/CHN/SX/2019/003/G9P8 MT10824/RVA/Hman-1CHNSX2019/339G9P8 MT170824RAHAT/39/G9P8 MT108 MT710815RVA/Hun MT710763/RVA/Human-wt/CHN/SX/2016/079/G9P8 MT710779/RVA/Human-wt/CHN/SX/2017/138/G9P8 MT710802/RVA/Human-wt/CHN/SX/2018/117/G9P8 MT710819/RVA/Human-wt/CHN/SX/2019/101/G9P8 - MT710792/RVA/Human-wt/CHN/SX/2018/004/G9P8

A LC477377.1 Human rotavirus A Human-wt/JPN/Tokyo18-43/2018/G9P8-E2 MT710808/RVA/Human-wt/CHN/SX/2018/391/G9P8 - MT710818/RVA/Human-wt/CHN/SX/2019/063/G9P8

88 MT710774/RVA/Human-wt/CHN/SX/2017/053/G9P8

68 MTOTH MT10795VAHUM MT710795/RVA/HUM WW/CHN/SX2018/064/G9P8 MT10765RVAHUman-W/CHN/SX2016/339/G9P8 MT710773/RVA/Human-wt/CHN/SX2017/050/G9P8 -MT710768/RVA/Human-wt/CHN/SX/2017/004/G9P8 MT710787/RVA/Human-wt/CHN/SX/2017/456/G9P8 MT710756/RVA/Human-wt/CHN/SX/2015/151/G9P8 MT710751/RVA/Human-wt/CHN/SX/2015/121/G9P8 MT710761/RVA/Human-wt/CHN/SX/2016/067/G9P8 MT710771/RVA/Human-wt/CHN/SX/2017/046/G9P8

KF673482.1 Human rotavirus A isolate BJ-0794 (1070)

KF673479.1 Human W/CHNSX2015/087/G9P8

A KF673479.1 Human rotanrus A isolate BJ-Q322

MT710755/RVA/Human-wt/CHN/SX/2015/143/G9P8

(16/112/G9P8 MT710745/RVA/Human-wt/CHN/SX/2015/061/G9P8

99 - MT710746/RVA/Human-wt/CHN/SX/2015/065/G9P8

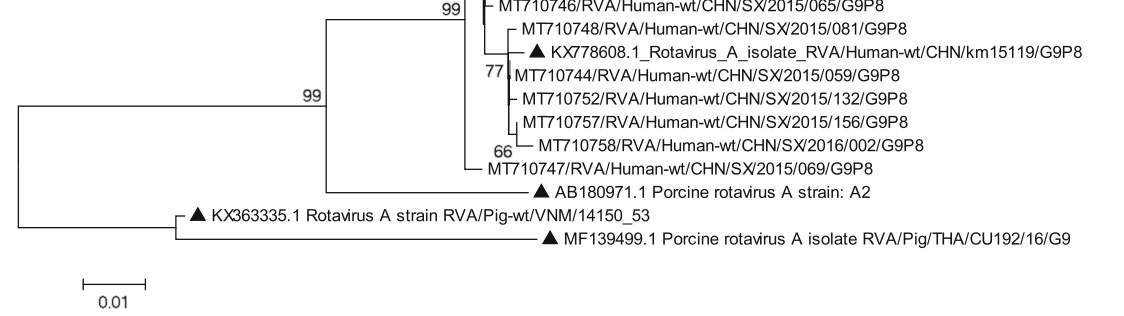

Fig. 6 Neighbor-joining phylogenetic tree constructed from the partial VP7 genes (798 bp) of G9 strains and representative RVA strains with the kimura-2-parameter model in MEGA program 5.0. Bootstrap values estimated with 1000 replicate data sets were indicated at each node. The scale bar indicated the number of nucleotide substitutions per site. Bootstrap values lower than $60 \%$ are not shown. The reference strains are represented by the triangle. The RVA strains sequenced in this study are represented by Accession number/Country/Province/Year/Serial number/Genotype

G1P[8] was still the predominant genotype among infants and children during 2012-2016 [27]. In 2014, G Ianiro reported that G1P[8] and G9P[8] strains were detected frequently from 2012 to 2014 in Italy, whereas G12P[8] caused a single large nosocomial outbreak [32].
These findings suggest that the G1, G2, and G3 strains, which represented the most prevalent genotypes worldwide in the past, are no longer the dominant strains in China. Besides, 24 strains (13.1\%, 24/183) could not be genotyped (VP7) using the primers (Beg9 and End9). 


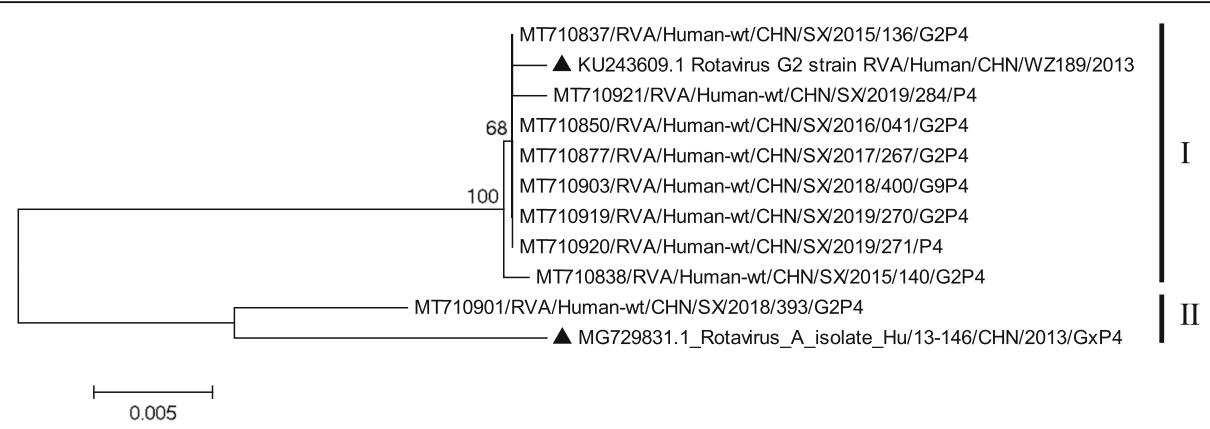

Fig. 7 Neighbor-joining phylogenetic tree constructed from the partial VP4 genes (696 bp) of P[4] strains and representative RVA strains with the kimura-2-parameter model in MEGA program 5.0. Bootstrap values estimated with 1000 replicate data sets were indicated at each node. The scale bar indicated the number of nucleotide substitutions per site. Bootstrap values lower than $60 \%$ are not shown. The reference strains are represented by the triangle. The RVA strains sequenced in this study are represented by Accession number/Country/Province/Year/Serial number/ Genotype

There may be genetic mutations related to these strains, and the whole genome sequencing is needed for sequence analysis.

The behavioral characteristics and spatial transition of the floating population are important factors in RVA transmission. Taiyuan, the capital city of Shanxi Province, has the largest inhabitants and mobile residents, also contribute to the largest number of RVA-positive cases and a variety of genotypes. Cities adjacent to Taiyuan, such as Xinzhou, Jinzhong, and Lvliang, showed similar genotype distribution, but the genotype diversity was significantly lower than that in Taiyuan. In contrast, some cities or regions, with a small population and low mobility, have fewer kinds of genotypes. For example, only two genotypes were detected in Yangquan, and G9P [8] was the only genotype that was detected after 2015 (Fig. 4, Yangquan). Besides, based on relatively few case statistics, our study found that the RVA genetic diversity in the north part of Shanxi was significantly less than that in the south, which may be related to the population density and economic development level.

Phylogenetic trees were used to represent the evolutionary history and diversification of species. Our results indicate that most RVA strains circulating in Shanxi Province have high genetic homology with those isolated from other provinces in China. Worldwide, although the dominant genotypes may be different in the same period, the Shanxi strains also maintain high genetic similarity with those isolated from China's neighboring countries, such as Japan, Thailand, and Pakistan. These results indicate that the genetic evolution of RVA strains occurs synchronously in certain areas, especially those areas with frequent population shifts.

Up to now, more than 20 genotypes have been identified from animals [4]. Our study found the Shanxi G3 strains were in the same evolutionary branch with two pig strains (CU140 and P50) isolated from Thailand and Slovenia, and the Shanxi G9 strains were in the same evolutionary branch with a pig strain (A2) isolated from the USA (Figs. 5, 6). These results imply that the Shanxi strains may have a common ancestor with the porcine rotavirus. However, it must be admitted that there are fewer rotavirus strains found in animals, so these conclusions may be overturned by in-depth research with the discovery of more rotavirus strains in animals.

Phylogenetic trees in this study were also used to evaluate the match between vaccine strains and Shanxi strains. Currently, only two rotavirus vaccines, LuoTeWei (G10P[12]) and RotaTeq (G1-G4, and P1a[8]), were licensed in China. Both vaccines have different genome compositions and antigenic characteristics, also provide broad heterotypic protection against other less common genotypes [33]. So far, all of them have not yet been included in the China National Immunization Program, and there were less available inoculation rate data reported officially. In 2018, Liu et al. investigated the rotavirus vaccination status of infants and young children born from 2008 to 2012 in six China's provinces (Jiangsu, Chongqing, Guangdong, Jiangxi, Heilongjiang, and Gansu), and found the proportions of the first and the second dose of rotavirus vaccination (LuoTeWei) were 32.8 and 9.7\%; Among the children vaccinated with the first dose, the vaccination age mainly concentrated in 2 to 21 months, of which the peak was 5 to 13 months [34]. Another study was conducted in Guangdong Province in 2013, He et al. reported an overall LuoTeWei vaccine coverage of 25.3\% among children aged 2-59 months [35].

Our results confirmed that the vaccine genotypes G4, G10, and P[12] of the LuoTeWei and RotaTeq were not found in Shanxi Province. Beyond that, the G1, G2, G3, and $P[8]$ strains in this study were related to the RotaTeq vaccine strains: RotaTeq-WI79-9(G1), RotaTeq-SC29(G2), RotaTeq-WI78-8(G3), and RotaTeq-WI79-4 (P1a[8]), respectively. However, there were about $7-12 \%$ differences in nucleotide and $3-11 \%$ in amino acid between the RotaTeq vaccine strain and Shanxi strains 


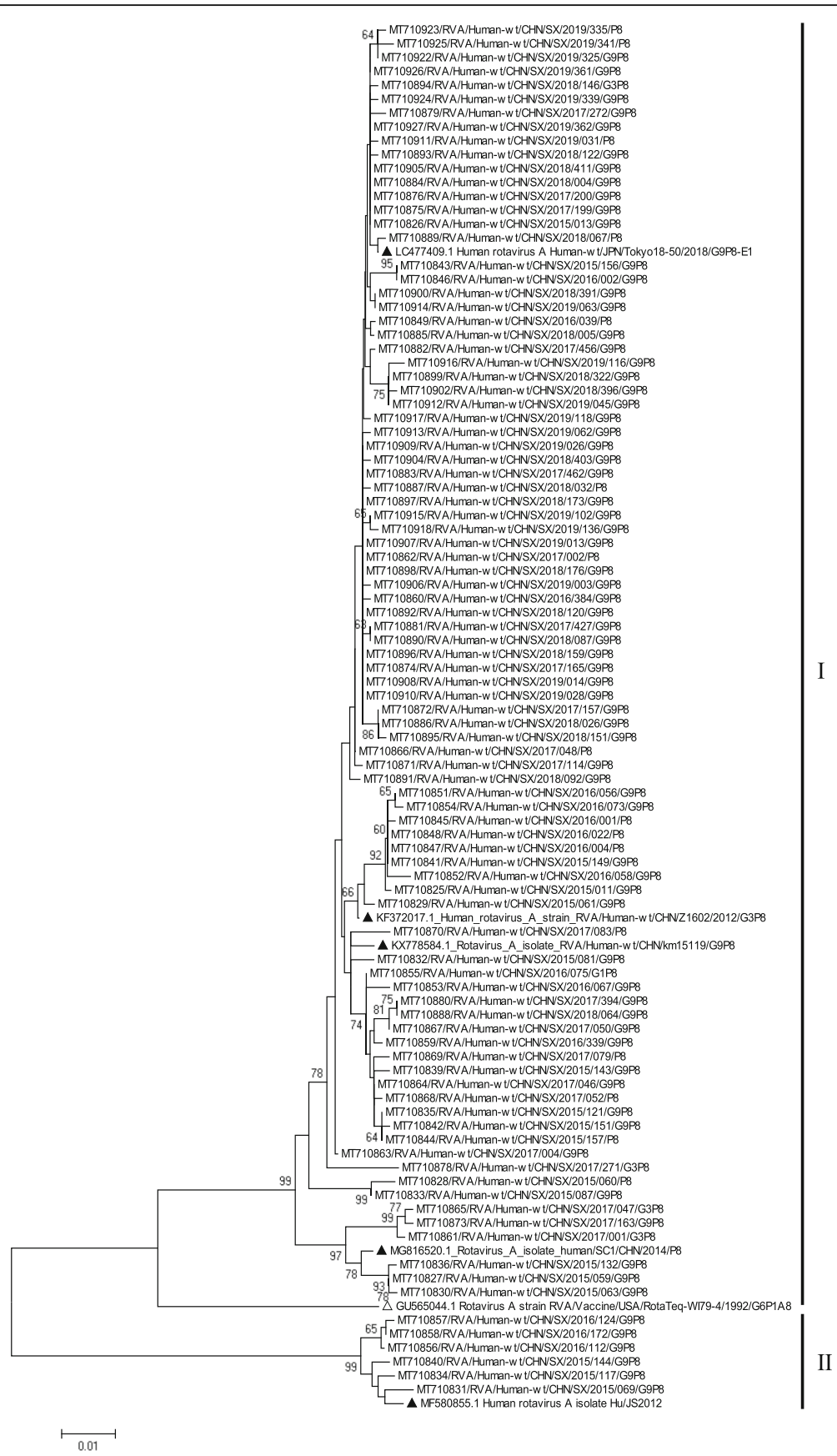

Fig. 8 Neighbor-joining phylogenetic tree constructed from the partial VP4 genes (696 bp) of P[8] strains and representative RVA strains with the kimura-2-parameter model in MEGA program 5.0. Bootstrap values estimated with 1000 replicate data sets were indicated at each node. The scale bar indicated the number of nucleotide substitutions per site. Bootstrap values lower than $60 \%$ are not shown. The reference strains are represented by the triangle. The RVA strains sequenced in this study are represented by Accession number/Country/Province/Year/Serial number/ Genotype

detected in this study. Our results also show that G9P [8] strains, which accounted for the highest proportion each year, had not been effectively controlled by the existing vaccines. The reason for this phenomenon may be the circulating strains cannot be effectively immunized by existing vaccines, or low inoculation rates. However, based on
Merck's data, RotaTeq is effective in the prevention of G9 rotavirus in a European study [33]. Due to the RotaTeq has been used locally for a short time, our results may not be used as a basis for evaluating the effectiveness.

This study has some limitations. First, only inpatients who have severe symptoms and need to be hospitalized 
for gastroenteritis were included in this study, the outpatients and those visiting the emergency department were not included. The positivity and genotypes of RVA apparently can be applied only to those with relatively high severity. Second, the effectiveness of a vaccine cannot be evaluated only by analyzing whether the genotype of the rotavirus vaccine matches the locally circulating strains. Furthermore, long-term ongoing surveillance is critical for evaluating interventions for RVA infections prevention and the use of phylogenetic analysis will be important to provide further insight into the impact of rotavirus vaccines on strain diversity.

\section{Conclusions}

This study highlights that the rotavirus G9P [8] strains have been predominantly circulating locally for a long time in Shanxi Province, China. Although it provides only the hospitalized children epidemiological data, it will provide comprehensive knowledge to the public health authorities to calculate the RVA related disease burden and assess the effectiveness of the rotavirus vaccine on these emerged RVA strains in China.

\section{Supplementary Information}

The online version contains supplementary material available at https://doi. org/10.1186/s12879-021-05795-4

Additional file 1: Table S1. Coinfections in inpatients with RVGE. Table S2. GenBank accession numbers assigned for all rotavirus genotypes based on gene segment VP4 and VP7 sequenced in this study.

\section{Abbreviations}

RVA: Group A rotavirus; RVGE: Rotavirus gastroenteritis; CHS: Children's Hospital of Shanxi; CDC: Center for Disease Control and Prevention; qRTPCR: Real-time quantitative reverse transcription polymerase chain reaction

\section{Acknowledgments}

The authors wish to thank all staff of Prof. Jianguo Xu's State Key Laboratory for Infectious Disease Prevention and Control, China CDC.

\section{Authors' contributions}

JW developed the original idea and reviewed the manuscript. LZ contributed to the development of the protocol, analyzed the data, and wrote the manuscript. XS contributed to the sample collection. $Y L, L L, X G, X Z, R G$, and LG contributed to the rotavirus strain collection. RT analyzed and interpreted the patient data regarding the rotavirus gastroenteritis. DM and JG contributed to the development of the protocol and provided technical support. All authors read and approved the final manuscript.

\section{Funding}

This work was supported by the grant 2015SKLID304 from the State Key Laboratory for Infectious Disease Prevention and Control, China CDC, the People's Republic of China. The funding agencies had no role in the design or analysis of the study.

\section{Availability of data and materials}

The datasets used and analyzed during the current study are available from the first author and the corresponding author on reasonable request.

\section{Ethics approval and consent to participate}

This study was approved by the Institutional Review Board and Human Research Ethics Committee of the Taiyuan Center for Disease Prevention and
Control, and the written consent was obtained. The children were enrolled after informed and verbal consent was obtained from the parents or guardians. Although the option of written approval was also provided, all participants chose to give their verbal consent due to convenience. Tests were performed on existing stool samples, and no additional tests involving any invasive procedures, were performed on patients. These procedures were approved by the above ethics committee.

Consent for publication

Not applicable.

\section{Competing interests}

No competing interests.

\section{Author details}

'Department of Microbiology Test, Taiyuan Center for Disease Control and Prevention, NO. 89 Xinjian South Road, Taiyuan 030012, Shanxi Province, China. ${ }^{2}$ Department of Disease Prevention and Public Health, Children's Hospital of Shanxi, Taiyuan 030001, Shanxi Province, China. ${ }^{3}$ Department of Tuberculosis Control and Prevention, Taiyuan Center for Disease Control and Prevention, Taiyuan 030012, Shanxi Province, China.

Received: 14 November 2020 Accepted: 12 January 2021

Published online: 21 January 2021

\section{References}

1. Troeger C, Forouzanfar M, Rao PC, Khalil I, Brown A, Reiner RC, et al. Estimates of global, regional, and national morbidity, mortality, and aetiologies of diarrhoeal diseases: a systematic analysis for the Global Burden of Disease Study 2015. Lancet Infect Dis. 2017:17(9):909-48.

2. Troeger C, Khalil IA, Rao PC, Cao S, Blacker BF, Ahmed T, et al. Rotavirus vaccination and the global burden of rotavirus diarrhea among children younger than 5 years. JAMA Pediatr. 2018;172(10):958-65.

3. Zhang J, Duan Z, Payne DC, Yen C, Pan X, Chang Z, et al. Rotavirus-specific and overall diarrhea mortality in Chinese children younger than 5 years 2003 to 2012. Pediatr Infect Dis J. 2015;34(10):e233-7.

4. Tuanthap S, Vongpunsawad S, Luengyosluechakul S, Sakkaew P, Theamboonlers A, Amonsin A, et al. Genome constellations of 24 porcine rotavirus group a strains circulating on commercial Thai swine farms between 2011 and 2016. PLoS One. 2019;14:e0211002.

5. Esposito S, Camilloni B, Bianchini S, laniro G, Polinori I, Farinelli E, et al. First detection of a reassortant G3P[8] rotavirus a strain in Italy: a case report in an 8-year-old child. Virol J. 2019:16:1-7.

6. Vrdoljak M, Gužvinec M, Trkulja V, Butić I, Ivić I, Krželj V, et al. Distribution of rotavirus genotypes in three Croatian regions among children $\leq 5$ years of age (2012-2014). Int J Infect Dis. 2019;89:3-9.

7. Crawford SE, Ramani S, Tate JE, Parashar UD, Svensson L, Hagbom M, et al. Rotavirus infection. Nat Rev Dis Primers. 2017;3:17083.

8. Shen $\mathrm{H}$, Zhang J, Li Y, Xie S, Jiang Y, Wu Y, et al. The 12 gastrointestinal pathogens spectrum of acute infectious diarrhea in a sentinel hospital, Shenzhen, China. Front Microbiol. 2016;7:1926.

9. Yamamoto SP, Kaida A, Kubo H, Iritani N. Gastroenteritis outbreaks caused by a DS-1-like G1P[8] Rotavirus strain, Japan, 2012-2013. Emerg Infect Dis. 2014:20(6):1030-3.

10. Kotirum S, Vutipongsatorn N, Kongpakwattana K, Hutubessy R, Chaiyakunapruk N. Global economic evaluations of rotavirus vaccines: a systematic review. Vaccine. 2017;35(26):3364-86.

11. Wu D, Yen C, Yin ZD, Li YX, Liu N, Liu YM, et al. The public health burden of rotavirus disease in children younger than five years and considerations for rotavirus vaccine introduction in China. Pediatr Infect Dis J. 2016;35(12): e392-8.

12. Zhang SX, Yang CL, Gu WP, Ai L, Serrano E, Yang P, et al. Case-control study of diarrheal disease etiology in individuals over 5 years in Southwest China. Gut Pathogens. 2016:8:58.

13. Zeng $Y$, Li T, Zhao B, Lai F, Tang $X$, Qiao $Y$, et al. Molecular epidemiology of group $A$ rotavirus in outpatient diarrhea infants and children in Chongqing, China, 2011-2015. J Med Virol. 2019:91(10):1788-96.

14. Fu C, Tate JE, Jiang B. Effectiveness of Lanzhou lamb rotavirus vaccine against hospitalized gastroenteritis: further analysis and update. Human Vaccines. 2010;6(11):953. 
15. Tian Y, Chughtai AA, Gao Z, Yan H, Chen Y, Liu B, et al. Prevalence and genotypes of group a rotavirus among outpatient children under five years old with diarrhea in Beijing, China, 2011-2016. BMC Infect Dis. 2018;18:1-11.

16. Fang ZY, Yang H, Qi J, Zhang J, Sun LW, Tang JY, et al. Diversity of rotavirus strains among children with acute diarrhea in China: 1998-2000 Surveillance study. J Clin Microbiol. 2002;40(5):1875-8.

17. Xu C, Fu J, Ai J, Zhang J, Liu C, Huo X, et al. Phylogenetic analysis of human G9P[8] rotavirus strains circulating in Jiangsu, China between 2010 and 2016. J Med Virol. 2018;90(9):1461-70.

18. Zhang $H$, Zhang Z, Wang $Y$, Wang $X$, Xia M, Wu H. Isolation, molecular characterization and evaluation of the pathogenicity of a porcine rotavirus isolated from Jiangsu Province, China. Arch Virol. 2015;160(5):1333-8.

19. Cai Y, Yin W, Zhou Y, Li B, Ai L, Pan M, et al. Molecular detection of porcine astrovirus in Sichuan Province, China. Virol J. 2016;13:6.

20. Zhao L, Xiong Y, Meng D, Guo J, Li Y, Liang L, et al. An 11-year study of shigellosis and Shigella species in Taiyuan, China: active surveillance, epidemic characteristics, and molecular serotyping. J Infect Public Health. 2017;10:794-8.

21. Parashar UD. Rotavirus Vaccines. In: The Vaccine Book: Second Edition; 2016

22. Jiang V, Jiang B, Tate J, Parashar UD, Patel MM. Performance of rotavirus vaccines in developed and developing countries. Hum Vaccin. 2010;6(7): $532-42$

23. Kirkwood CD, Ma LF, Carey ME, Steele AD. The rotavirus vaccine development pipeline. Vaccine. 2019;37(50):7328-35.

24. Jia L, Lin C, Gao Z, Qu M, Yang J, Sun J, et al. Prevalence and factors associated with different pathogens of acute diarrhea in adults in Beijing, China. J Infect Dev Ctries. 2016;10(11):1200-7.

25. Chen YH, Chen F, Zhou T, Chen JY, Zheng TL, Xu X, et al. Prevalence and clinical profile of rotavirus a infection among diarrhoeal children and phylogenetic analysis with vaccine strains in Chengdu, West China, 20092014. Trop Med Int Health. 2018;23(7):704-13.

26. Aliabadi N, Antoni S, Mwenda JM, Weldegebriel G, Biey JNM, Cheikh D, et al. Global impact of rotavirus vaccine introduction on rotavirus hospitalisations among children under 5 years of age, 2008-16: findings from the global rotavirus surveillance network. Lancet Glob Health. 2019:7(7):e893-903.

27. Giri S, Nair NP, Mathew A, Manohar B, Simon A, Singh T, et al. Rotavirus gastroenteritis in Indian children $<5$ years hospitalized for diarrhoea, 2012 to 2016. BMC Public Health. 2019;19:1-10.

28. Wang G, Shen Z, Qian F, Li Y, Yuan Z, Zhang J. Genetic diversity of sapovirus in non-hospitalized adults with sporadic cases of acute gastroenteritis in Shanghai, China. J Clin Virol. 2014;59(4):250-4.

29. Yu J, Lai S, Geng Q, Ye C, Zhang Z, Zheng Y, et al. Prevalence of rotavirus and rapid changes in circulating rotavirus strains among children with acute diarrhea in China, 2009-2015. J Infect. 2019:78(1):66-74.

30. Sadiq A, Bostan N, Bokhari H, Matthijnssens J, Yinda KC, Raza S, et al. Molecular characterization of human group a rotavirus genotypes circulating in Rawalpindi, Islamabad, Pakistan during 2015-2016. PLoS One. 2019;14:1-28.

31. Agbla JMM, Capo-Chichi A, Agbankpé AJ, Dougnon TV, Yadouleton AWM, Houngbégnon $\mathrm{O}$, et al. Epidemiological survey of rotaviruses responsible for infantile diarrhea by the Immunomolecular technique in Cotonou (Benin, West Africa). Int J Microbiol. 2018;2018:3602967.

32. Ianiro G, Delogu R, Fiore L, Monini M, Ruggeri FM, Pagani E, et al. Group a rotavirus genotypes in hospital-acquired gastroenteritis in Italy, 2012-14. J Hosp Infect. 2017;96(3):262-7.

33. Plosker GL. Pentavalent rotavirus vaccine (RotaTeq ${ }^{\circledR}$ ): a review of its use in the prevention of rotavirus gastroenteritis in Europe. Drugs. 2010;70(9): 1165-88.

34. Liu Y, Yue CY, Li Y, Wang YM, Gao SR, Wang ZG, et al. Analysis of vaccination situation of orial live attenuated rotavirus vaccine (LLR strain) among children in 6 provinces of China. Zhonghua Yu Fang Yi Xue Za Zhi. 2018;52(3):282-6.

35. He Q, Wang $\mathrm{M}$, Xu J, Zhang C, Wang $H$, Zhu W, et al. Rotavirus vaccination coverage among children aged 2-59 months: a report from Guangzhou, China. PLoS ONE. 2013;8(6):e68169.

\section{Publisher's Note}

Springer Nature remains neutral with regard to jurisdictional claims in published maps and institutional affiliations.

\section{Ready to submit your research? Choose BMC and benefit from:}

- fast, convenient online submission

- thorough peer review by experienced researchers in your field

- rapid publication on acceptance

- support for research data, including large and complex data types

- gold Open Access which fosters wider collaboration and increased citations

- maximum visibility for your research: over $100 \mathrm{M}$ website views per year

At BMC, research is always in progress.

Learn more biomedcentral.com/submissions 